Research Article

\title{
Submanifolds of Sasakian Manifolds with Certain Parallel Operators
}

\author{
Amira A. Ishan \\ Department of Mathematics, Taif University, P.O. Box 20314, Makkah 21599, Saudi Arabia \\ Correspondence should be addressed to Amira A. Ishan; amiraishan@hotmail.com
}

Received 19 November 2013; Accepted 22 December 2013; Published 2 February 2014

Academic Editors: T. Friedrich and I. Strachan

Copyright (C) 2014 Amira A. Ishan. This is an open access article distributed under the Creative Commons Attribution License, which permits unrestricted use, distribution, and reproduction in any medium, provided the original work is properly cited.

We study submanifolds of Sasakian manifolds and obtain a condition under which certain naturally defined symmetric tensor field on the submanifold is to be parallel and use this result to obtain conditions under which a submanifold of the Sasakian manifold is an invariant submanifold.

\section{Introduction}

Differential geometry of submanifolds of Sasakian manifold is an interesting branch and has been subject of investigations of many mathematicians. There are four important types of submanifolds of a Sasakian manifold, namely, invariant submanifolds, anti-invariant submanifolds, CR submanifolds, and slant submanifolds. All these types of submanifolds have been studied quite extensively; for invariant and antiinvariant submanifolds one can refer to [1-5] and for slant submanifolds one can refer to [6-8] and references therein. A submanifold $M$ of a Sasakian manifold $(\bar{M}, \varphi, \xi, \eta, g)$ is said to be a CR-submanifold if there is a pair of orthogonal complementary distributions $\mathscr{D}$ and $\mathscr{D}^{\perp}$ defined on $M$ of which $\mathscr{D}$ is invariant under $\varphi$ and $\mathscr{D}^{\perp}$ is totally real distribution so that $\varphi \mathscr{D}^{\perp}$ is subbundle of the normal bundle. CR submanifolds of a Sasakian manifold have been studied in [9-13] getting various geometric properties of the CRsubmanifold. A submanifold of a Sasakian manifold naturally carries four operators, $\phi, F, \psi$, and $G$, defined on this submanifold. In [14], it has been shown that a submanifold of a Sasakian manifold with parallel $\phi$ is essentially a CRsubmanifold. In this paper, we are interested in finding conditions under which a submanifold of a Sasakian manifold with parallel operator $F$ is an invariant submanifold. First we show the existence of a symmetric tensor field $C$ on any submanifold of a Sasakian manifold and study its properties and use these properties to obtain conditions under which a submanifold is an invariant submanifold (cf. Theorem 5).

\section{Preliminaries}

Let $(\bar{M}, \varphi, \xi, \eta, g)$ be a $(2 m+1)$-dimensional almost contact metric manifold. Then the structure tensor satisfies (cf. [15])

$$
\begin{gathered}
\varphi^{2}=-I+\eta \otimes \xi, \quad \eta(\xi)=1, \\
\varphi \xi=0, \quad \eta \circ \varphi=0, \\
g(\varphi X, \varphi Y)=g(X, Y)-\eta(X) \eta(Y), \\
g(X, \xi)=\eta(X),
\end{gathered}
$$

where $X$ and $Y$ are smooth vectors fields on $\bar{M}$. An almost contact metric manifold $(\bar{M}, \varphi, \xi, \eta, g)$ is said to be a Sasakian manifold if it satisfies

$$
\left(\bar{\nabla}_{X} \varphi\right)(Y)=g(X, Y) \xi-\eta(Y) X, \quad \bar{\nabla}_{X} \xi=-\varphi X,
$$

for smooth vector fields $X$ and $Y$, where $\bar{\nabla}$ is the Riemannian connection on $\bar{M}$.

Let $M$ be an $n$-dimensional submanifold of the Sasakian manifold $(\bar{M}, \varphi, \xi, \eta, g)$. Denote by the same $g$ the Riemannian metric induced on the submanifold $M$ and by $\Gamma\left(T^{\perp} M\right)$ the space of smooth sections of the normal bundle $T^{\perp} M$ of $M$. Then we define operators $\phi, F, \psi$, and $G$ as follows:

$$
\varphi X=\phi X+F X, \quad \varphi N=\psi N+G N,
$$

where $X \in \chi(M)$ is the Lie algebra of smooth vector fields on $M$ and $N \in \Gamma\left(T^{\perp} M\right) ; \phi X$ (resp., $\left.F X\right)$ denotes the tangent part 
(resp., normal part) of $\varphi X$; and $\psi N$ (resp., GN) denotes the tangent part (resp., normal part) of $\varphi N$.

Also for the structure vector field $\xi$ of the Sasakian manifold $\bar{M}$, we set

$$
\xi=u+t
$$

where $u \in \chi(M)$ and $t \in \Gamma\left(T^{\perp} M\right)$. Define a smooth one form $\alpha$ on the submanifold $M$ and $\beta: \Gamma\left(T^{\perp} M\right) \rightarrow C^{\infty}(M)$ by $\alpha(X)=g(X, u), X \in \chi(X)$, and $\beta(N)=g(N, t), N \in$ $\Gamma\left(T^{\perp} M\right)$. It can be easily checked that the operators $\phi, F, \psi$, and $G$ satisfied the relations

$$
\begin{gathered}
g(\phi X, Y)=-g(X, \phi Y), \quad X, Y \in \chi(M), \\
g\left(G N_{1}, N_{2}\right)=-g\left(N_{1}, G N_{2}\right), \quad N_{1}, N_{2} \in \Gamma\left(T^{\perp} M\right), \\
g(F X, N)=-g(X, \psi N), \quad X \in \chi(M), \quad N \in \Gamma\left(T^{\perp} M\right),
\end{gathered}
$$$$
\phi^{2} X+\psi(F X)=-X+\alpha(X) u,
$$$$
F(\phi X)=-G(F X)+\alpha(X) t, \quad X \in \chi(M),
$$$$
G^{2} N+F(\psi N)=-N+\beta(N) t,
$$$$
\phi(\psi(N))=-\psi(G N)+\beta(N) u, \quad N \in \Gamma\left(T^{\perp} M\right),
$$$$
\phi u=-\psi t, \quad F u=-G t .
$$

Also for the submanifold $M$, we have the following Gauss and Weingarten formulas (cf. [16]):

$$
\begin{array}{ll}
\bar{\nabla}_{X} Y=\nabla_{X} Y+h(X, Y), & X, Y \in \chi(M), \\
\bar{\nabla}_{X} N=-A_{N} X+\nabla_{X}^{\perp} N, & N \in \Gamma\left(T^{\perp} M\right),
\end{array}
$$

where $\nabla^{\perp}$ is the normal connection, $h$ is the second fundamental form and $A_{N}$ is the Weingarten map with respect to the normal bundle $T^{\perp} M$ of $M$ and we have

$$
\begin{gathered}
g\left(A_{N} X, Y\right)=g(h(X, Y), N), \\
X, Y \in \chi(M), \quad N \in \Gamma\left(T^{\perp} M\right) .
\end{gathered}
$$

For the operators $F$ and $\psi$ on the submanifold $M$ of the Sasakian manifold $\bar{M}$, we define the following covariant derivatives:

$$
\begin{gathered}
\left(D_{X} F\right) Y=\nabla_{X}^{\perp} F Y-F\left(\nabla_{X} Y\right), \quad X, Y \in \chi(M), \\
\left(D_{X} \psi\right) N=\nabla_{X} \psi N-\psi\left(\nabla_{X}^{\perp} N\right), \\
X \in \chi(M), \quad N \in \Gamma\left(T^{\perp} M\right) .
\end{gathered}
$$

Then using (2)-(4) and (11) we immediately get the following.
Lemma 1. Let $M$ be a submanifold of the Sasakian manifold $\bar{M}$. Then one has

$$
\begin{array}{r}
\nabla_{X} u=-\phi X+A_{t} X, \quad \nabla_{X}^{\perp} t=-h(X, u)-F X, \\
X \in \chi(M), \\
\left(\nabla_{X} \phi\right) Y=g(X, Y) u-\alpha(Y) X+A_{F Y} X \\
+\psi(h(X, Y)), \quad X, Y \in \chi(M), \\
\left(D_{X} F\right) Y=g(X, Y) t-h(X, \phi Y) \\
+G(h(X, Y)), \quad X, Y \in \chi(M), \\
\left(D_{X} \psi\right) N=-\beta(N) X+A_{G N} X-\phi\left(A_{N} X\right), \\
X \in \chi(M), \quad N \in \Gamma\left(T^{\perp} M\right), \\
\left(\nabla_{X}^{\perp} G\right) N=-F\left(A_{N} X\right)-h(X, \psi N), \\
X \in \chi(M), \quad N \in \Gamma\left(T^{\perp} M\right) .
\end{array}
$$

\section{Submanifolds with Parallel $F$}

In this section we will study a submanifold $M$ of the Sasakian manifold $(\bar{M}, \varphi, \xi, \eta, g)$ with structure vector field $\xi$ tangent to $M$ and the operator $F$ is parallel. Note that it is customary to require that $\xi$ is tangent to $M$ (cf. [13]), rather than normal as it is too restrictive, as in this case $M$ must be totally real [13, Proposition 1.1, page 43] or leads to too complicated embedding equations.

If we define the operators

$$
\begin{gathered}
B=\psi \circ F: \chi(M) \longrightarrow \chi(M), \\
C=F \circ \psi: \Gamma\left(T^{\perp} M\right) \longrightarrow \Gamma\left(T^{\perp} M\right),
\end{gathered}
$$

by using (7) it is easy to see that $B$ and $C$ are symmetric tensor fields.

In [17], Yano and Kon have studied the submanifolds of a Sasakian manifold with operator $F$ is parallel and have proved that these submanifolds are contact CR submanifolds [17, Proposition 3.3, page 241]. Thus all our results for submanifolds with parallel operator $F$ of a Sasakian manifold are automatically the results of contact CR submanifolds of the Sasakian manifold. First we will prove the following.

Theorem 2. Let $M$ be an n-dimensional submanifold of a $(2 m+1)$-dimensional Sasakian manifold $(\bar{M}, \varphi, \xi, \eta, g)$ with structure vector field $\xi$ tangent to $M$. If the operator $F$ is parallel, then $C$ is also parallel.

Proof. For $X \in \chi(M)$ and $N \in \Gamma\left(T^{\perp} M\right)$ we have

$$
\begin{aligned}
\nabla_{X}^{\perp}(C N) & =\nabla_{X}^{\perp} F(\psi(N)) \\
& =\left(D_{X} F\right)(\psi(N))+F\left(\nabla_{X} \psi(N)\right) \\
& =\left(D_{X} F\right)(\psi(N))+F\left(\left(D_{X} \psi\right) N\right)+F\left(\psi\left(\nabla_{X}^{\perp} N\right)\right) \\
& =\left(D_{X} F\right)(\psi(N))+F\left(\left(D_{X} \psi\right) N\right)+C\left(\nabla_{X}^{\perp} N\right),
\end{aligned}
$$


and consequently

$$
\left(\nabla_{X}^{\perp} C\right) N=\left(D_{X} F\right)(\psi(N))+F\left(\left(D_{X} \psi\right) N\right) .
$$

Using Lemma 1 in above equation and noting that $\beta(N)=$ $g(N, t)=0$, we get

$$
\begin{aligned}
\left(\nabla_{X}^{\perp} C\right) N= & -h(X, \phi(\psi(N)))+G(h(X, \psi(N))) \\
& +F\left(A_{G N} X-\phi\left(A_{N} X\right)\right) .
\end{aligned}
$$

Now since $F$ is parallel then by Lemma 1 with $t=0$, we get

$$
h(X, \phi Y)=G(h(X, Y)) \text {. }
$$

Also, using (6) we get

$$
g(h(X, \phi Y), N)=-g(h(X, Y), G N) ;
$$

by using the above equation and (12) we get

$$
A_{G N} X=\phi A_{N} X \text {. }
$$

Using (21) and (19) in (18), we conclude that $\left(\nabla_{X}^{\perp} C\right) N=0$ and this proves the theorem.

For $N \in \Gamma\left(T^{\perp} M\right)$, let $C(N)=\lambda N, \lambda \in C^{\infty}(M)$; that is, $N$ is the eigenvector of $C$ corresponding to an eigenvalue $\lambda$.

Lemma 3. Let $M$ be an $n$-dimensional submanifold of the Sasakian manifold $(\bar{M}, \varphi, \xi, \eta, g)$ with structure vector field $\xi$ tangent to $M$. If the operator $F$ is parallel, then the eigenvalues of $C$ are constant.

Proof. Let $C(N)=\lambda N, \lambda \in C^{\infty}(M), N \in \Gamma\left(T^{\perp} M\right)$. Without loss of generality we can assume that $N$ is a unit normal vector field. As $F$ is parallel then, by Theorem 2, we have $C$ being parallel and consequently

$$
\begin{aligned}
0 & =\left(\nabla_{X}^{\perp} C\right)(N)=\nabla_{X}^{\perp}(C N)-C\left(\nabla_{X}^{\perp} N\right) \\
& =X(\lambda) N+\lambda\left(\nabla_{X}^{\perp} N\right)-C\left(\nabla_{X}^{\perp} N\right) .
\end{aligned}
$$

Taking inner product with $N$, we get

$$
0=X(\lambda) \text {, }
$$

which proves that $\lambda$ is a constant.

For a local orthonormal frame $\left\{N_{1}, \ldots, N_{k}\right\}$ (where $2 m+$ $1=n+k)$ on $M$, we define smooth function $\|G\|: M \rightarrow R$ by

$$
\|G\|^{2}=\sum_{i=1}^{k} g\left(G\left(N_{i}\right), G\left(N_{i}\right)\right) ;
$$

then using (6), (7), and (9), we obtain

$$
\|G\|^{2}=k-\|\psi\|^{2},
$$

where $\|\psi\|^{2}=\sum_{i=1}^{k} g\left(\psi\left(N_{i}\right), \psi\left(N_{i}\right)\right)$. Also, we define

$$
\|C\|^{2}=\sum_{i=1}^{k} g\left(C\left(N_{i}\right), C\left(N_{i}\right)\right) ;
$$

since $C$ is symmetric we can choose an orthonormal frame $\left\{N_{1}, \ldots, N_{k}\right\}$ that diagonalizes $C$.
Lemma 4. Let $M$ be $n$-dimensional submanifold of the Sasakian manifold $(\bar{M}, \varphi, \xi, \eta, g)$ with structure vector field $\xi$ tangent to $M$. If the operator $F$ is parallel, then $\|\psi\|^{2}$ is a constant and $\|G\|^{2}+\|\psi\|^{2}=k$ holds; consequently $\|G\|^{2}$ is also a constant.

Proof. Suppose F is parallel; then by (25), we have

$$
\|G\|^{2}+\|\psi\|^{2}=k
$$

By (7) we have

$$
X\left(\|\psi\|^{2}\right)=-X\left(\sum_{i=1}^{k}\left(g\left(C\left(N_{i}\right), N_{i}\right)\right)\right) ;
$$

since the operator $C$ is symmetric we get

$$
X\left(\|\psi\|^{2}\right)=-\sum_{i=1}^{k}\left\{g\left(\left(\nabla_{X}^{\perp} C\right) N_{i}, N_{i}\right)+2 g\left(C\left(N_{i}\right), \nabla_{X}^{\perp} N_{i}\right)\right\}
$$

since by Theorem 2 since $F$ is parallel implies $C$ also parallel and the local orthonormal frame $\left\{N_{1}, \ldots, N_{k}\right\}$ on normal vector fields that diagonalizes $C$ with the equation $C\left(N_{i}\right)=$ $\lambda_{i} N_{i}$, thus we get that

$$
X\left(\|\psi\|^{2}\right)=-\sum_{i=1}^{k} \lambda_{i} X\left(g\left(N_{i}, N_{i}\right)\right)=0
$$

which proves that $\|\psi\|^{2}$ is a constant. Finally as $\|\psi\|^{2}$ is a constant, we get that $\|G\|^{2}$ is also a constant.

Note that the operator $C$ plays an important role in the geometry of submanifolds of a Sasakian manifold; for instance the following theorem shows that $\operatorname{tr} \cdot C=0$ implies that the submanifolds are invariant submanifolds.

Theorem 5. Let $M$ be an n-dimensional connected submanifold of the Sasakian manifold $(\bar{M}, \varphi, \xi, \eta, g)$ with structure vector field $\xi$ tangent to $M$. If $\operatorname{tr} \cdot C=0$, then $M$ is an invariant submanifold.

Proof. Suppose $\operatorname{tr} \cdot C=0$; then we have

$$
\begin{aligned}
0 & =\operatorname{tr} \cdot C=\sum_{i=1}^{k} g\left(N_{i}, C N_{i}\right) \\
& =-\sum_{i=1}^{k} g\left(\psi N_{i}, \psi N_{i}\right)=-\|\psi\|^{2},
\end{aligned}
$$

and that gives $\psi=0$. This also gives $B=0$ and consequently

$$
\begin{array}{r}
0=g(B X, X)=-g(F X, F X)=-\|F X\|^{2}, \\
X \in \chi(M) ;
\end{array}
$$

that is, $F X=0, X \in \chi(M)$. That is, $M$ is an invariant submanifold. 
Theorem 6. Let $M$ be an n-dimensional submanifold of the Sasakian manifold $(\bar{M}, \varphi, \xi, \eta, g)$ with structure vector field $\xi$ tangent to $M$. The operator $F$ is parallel if and only if $\psi$ is parallel.

Proof. If $F$ is parallel then by Lemma 1 with $t=0$ we get

$$
h(X, \phi Y)=G(h(X, Y)) .
$$

Taking inner product with $N \in \Gamma\left(T^{\perp} M\right)$ and using (6) we get

$$
g(h(X, \phi Y), N)=-g(h(X, Y), G N),
$$

and using (12) and (5) we get

$$
g\left(\phi\left(A_{N} X\right), Y\right)=g\left(A_{G N} X, Y\right),
$$

which implies that

$$
A_{G N} X=\phi\left(A_{N} X\right) .
$$

Now using the above equation in Lemma 1 and the fact that $t=0$ we get that $\beta(N)=0$ and $\left(D_{X} \psi\right) N=0$ which means that $\psi$ is parallel.

Conversely, if we suppose that the operator $\psi$ is parallel then by Lemma 1 with $t=0$, we get

$$
A_{G N} X=\phi\left(A_{N} X\right) .
$$

Also using (5) to get

$$
g\left(A_{G N} X, Y\right)=-g\left(A_{N} X, \phi Y\right),
$$

which together with (12) and (6) gives

$$
g(G(h(X, Y)), N)=g(h(X, \phi Y), N),
$$

we get that

$$
G(h(X, Y))=h(X, \phi Y),
$$

and using the above equation in Lemma 1 with $t=0$ we get $\left(D_{X} F\right)=0$ which proves that $F$ is parallel.

As a direct consequence of the above theorem and Proposition 3.3 in [17], we have the following.

Corollary 7. Let $M$ be a submanifold of a Sasakian manifold $(\bar{M}, \varphi, \xi, \eta, g)$. If $\psi$ is parallel, then $M$ is a contact CR-submanifold of $\bar{M}$.

\section{Conflict of Interests}

The author declares that there is no conflict of interests regarding the publication of this paper.

\section{References}

[1] I. Ishihara, "Anti-invariant submanifolds of a Sasakian space form," Kodai Mathematical Journal, vol. 2, no. 2, pp. 171-186, 1979.
[2] M. Kon, "On some invariant submanifolds of normal contact metric manifolds," Tensor, vol. 28, pp. 133-138, 1974.

[3] M. Kon, "Invariant submanifolds in Sasakian manifolds," Mathematische Annalen, vol. 219, no. 3, pp. 277-290, 1976.

[4] K. Yano and M. Kon, Anti-Invariant Submanifolds, vol. 21 of Lecture Notes in Pure and Applied Mathematics, Marcel Dekker, New York, NY, USA, 1976.

[5] K. Yano, M. Kon, and I. Ishihara, "Anti-invariant submanifolds with flat normal connection," Journal of Differential Geometry, vol. 13, no. 4, pp. 577-588, 1978.

[6] J. L. Cabrerizo, A. Carriazo, L. M. Fernández, and M. Fernández, "Semi-slant submanifolds of a Sasakian manifold," Geometriae Dedicata, vol. 78, no. 2, pp. 183-199, 1999.

[7] J. L. Cabrerizo, A. Carriazo, L. M. Fernández, and M. Fernández, "Slant submanifolds in Sasakian manifolds," Glasgow Mathematical Journal, vol. 42, no. 1, pp. 125-138, 2000.

[8] J. L. Cabrerizo, A. Carriazo, L. M. Fernández, and M. Fernández, "Structure on a slant submanifold of a contact manifold," Indian Journal of Pure and Applied Mathematics, vol. 31, no. 7, pp. 857$864,2000$.

[9] I. Hasegawa and I. Mihai, "Contact CR-warped product submanifolds in Sasakian manifolds," Geometriae Dedicata, vol. 102, pp. 143-150, 2003.

[10] K. Yano and M. Kon, "CR-sous-variétés d'un espace projectif complexe," Comptes Rendus Hebdomadaires des Séances de l'Académie des Sciences. Séries A et B, vol. 288, no. 9, pp. A515A517, 1979.

[11] K. Yano and M. Kon, "Differential geometry of CR-sub-manifolds," Geometriae Dedicata, vol. 10, no. 1-4, pp. 369-391, 1981.

[12] K. Yano and M. Kon, "Contact CR submanifolds," Kodai Mathematical Journal, vol. 5, no. 2, pp. 238-252, 1982.

[13] K. Yano and M. Kon, CR Submanifolds of Kaehlerian and Sasakian Manifolds, vol. 30 of Progress in Mathematics, Birkhäuser, Boston, Mass, USA, 1983.

[14] A. A. Ishan, "Submanifolds of a Sasakianmanifold," Submitted.

[15] D. E. Blair, Contact Manifolds in Riemannian Geometry, vol. 509 of Lecture Notes in Mathematics, Springer, Berlin, Germany, 1976.

[16] B.-Y. Chen, Total Mean Curvature and Submanifolds of Finite Type, vol. 1 of Series in Pure Mathematics, World Scientific, Singapore, 1984.

[17] K. Yano and M. Kon, "On contact CR submanifolds," Journal of the Korean Mathematical Society, vol. 26, no. 2, pp. 231-262, 1989. 


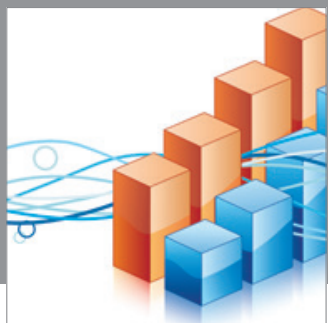

Advances in

Operations Research

mansans

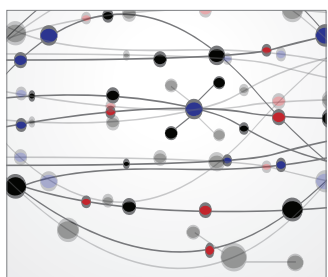

The Scientific World Journal
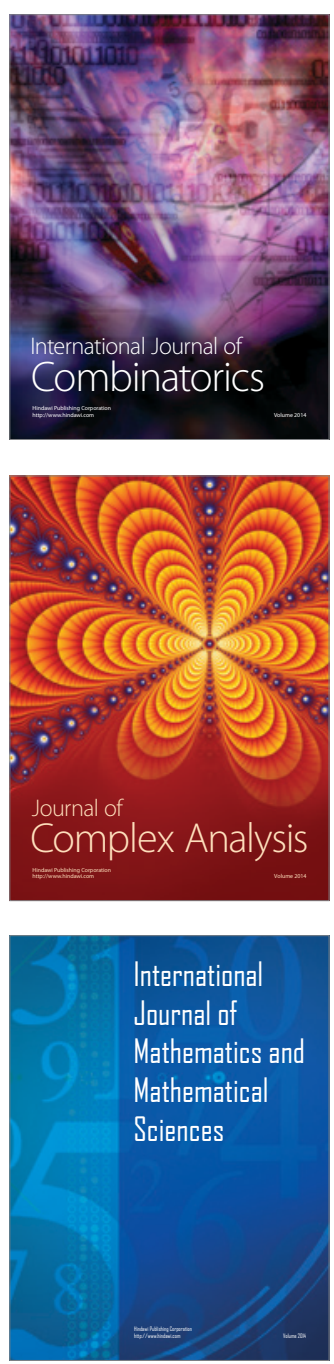
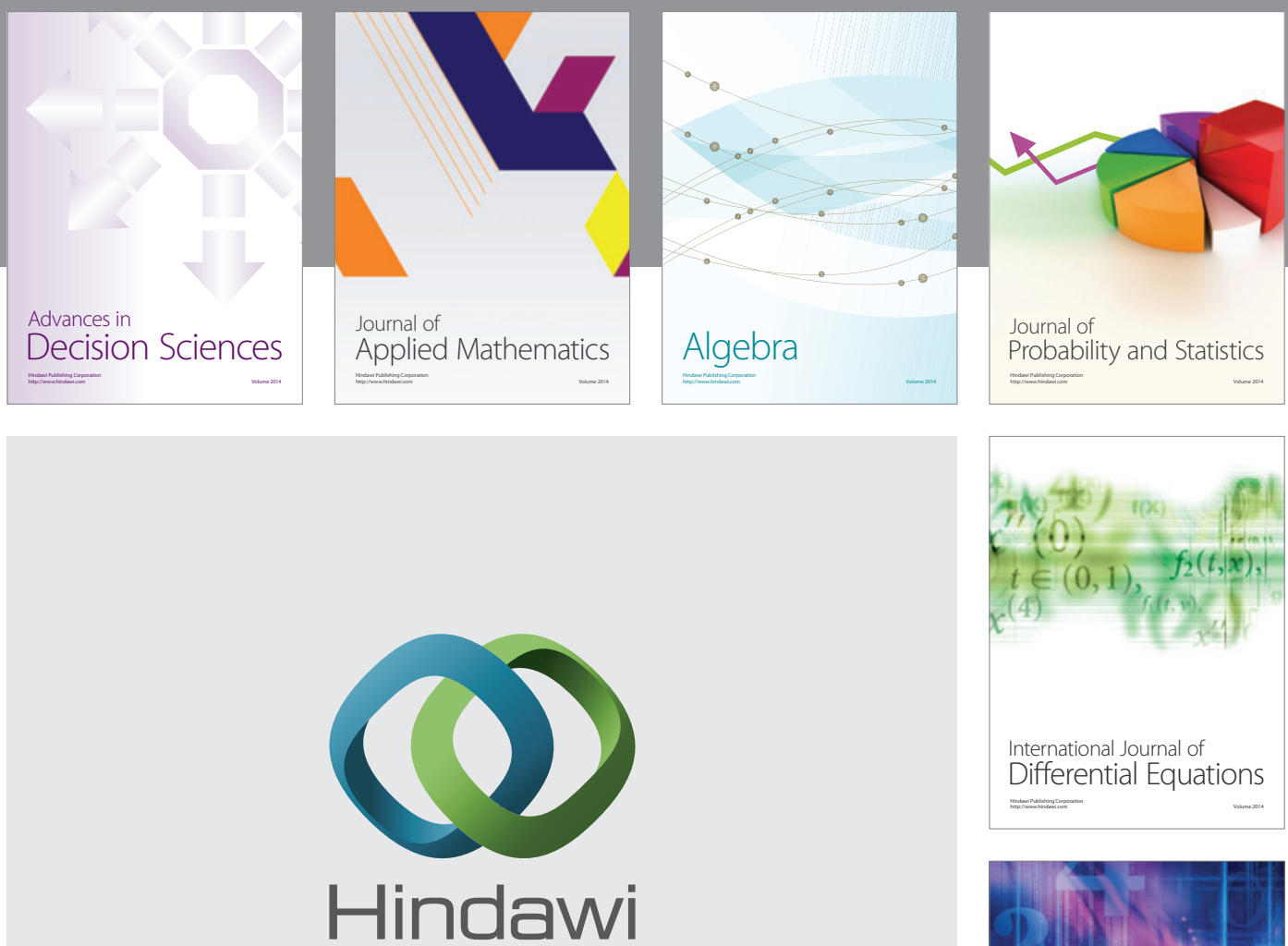

Submit your manuscripts at http://www.hindawi.com
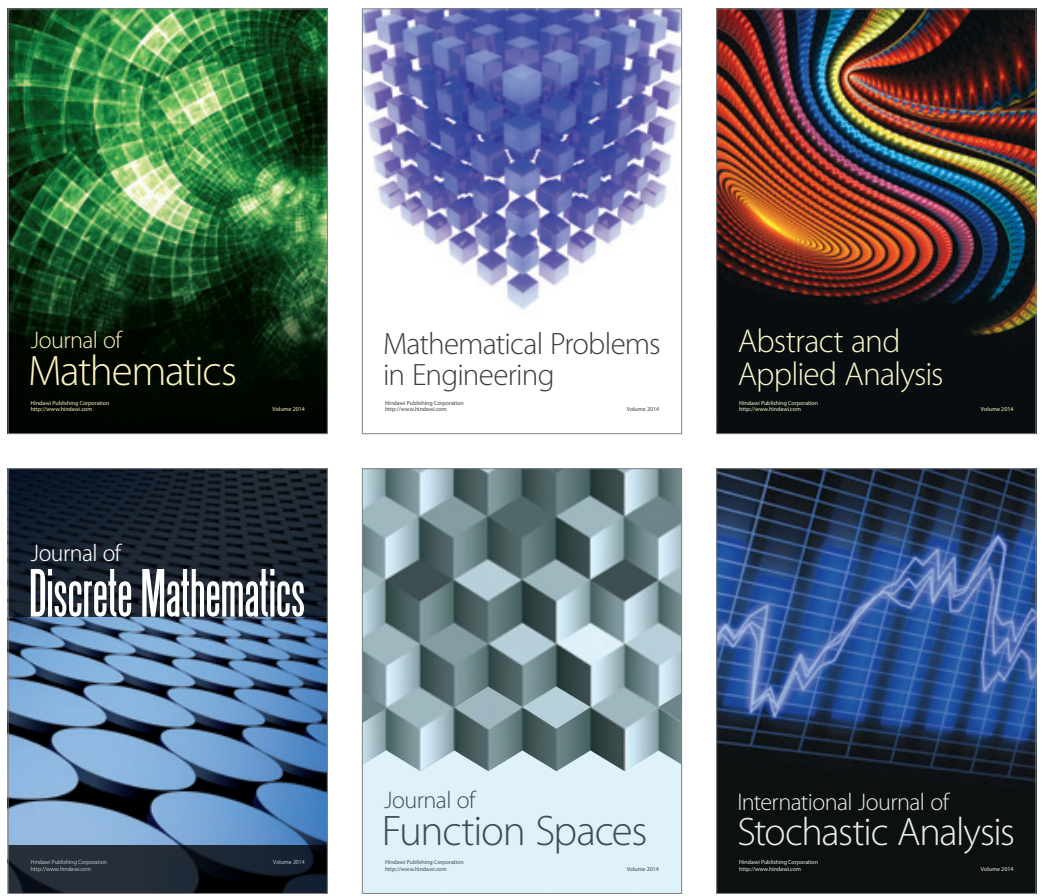

Journal of

Function Spaces

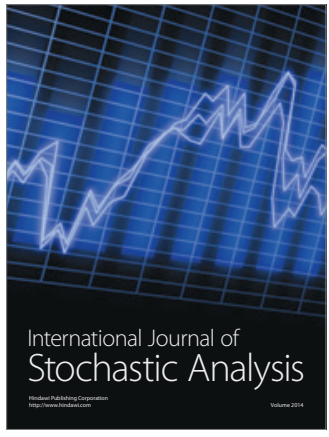

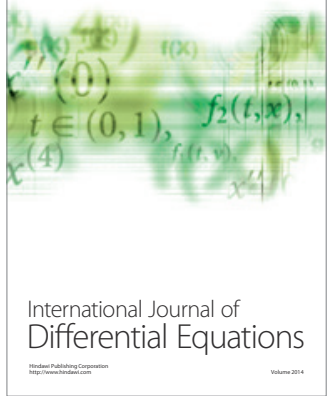
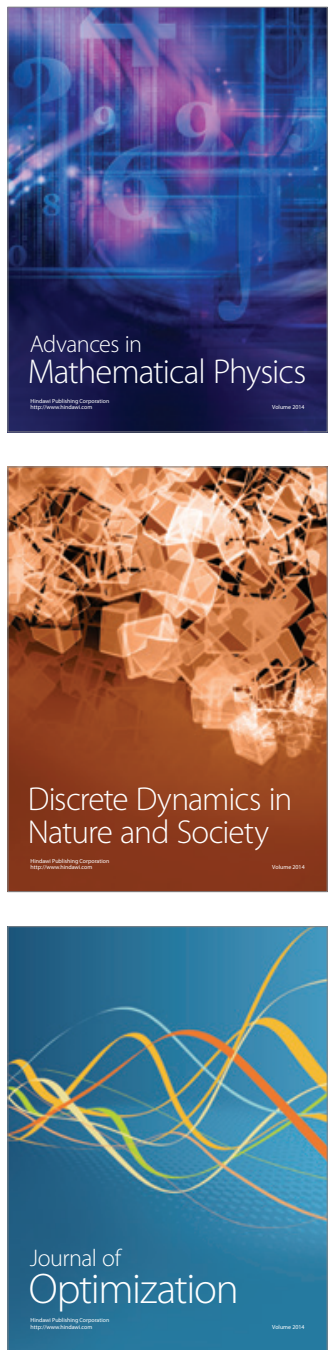